\title{
$\angle$ Research Square \\ Expression of ASPN with Prognostic Values in Gastric Cancer: A Study Based on TCGA Data
}

wen chao li ( $\square$ liwencha0301@163.com )

Chinese PLA General Hospital https://orcid.org/0000-0002-0308-070X

\section{Zhe Xu}

Chinese PLA General Hospital

\section{Hui Chen}

7th Medical Center of Chinese PLA General Hospital

\section{Zhen dong Wang}

7th Medical Center of Chinese PLA General Hospital

\section{Yang Xu}

Chinese PLA General Hospital

\section{Li Liu}

7th Medical Center of Chinese PLA General Hospital

Rui jiang Xu

Chinese PLA General Hospital

\section{Research Article}

Keywords: Asporin, Gastric cancer, Independent predictor, Prognosis, Biomarker

Posted Date: May 27th, 2021

DOl: https://doi.org/10.21203/rs.3.rs-550453/v1

License: (c) (1) This work is licensed under a Creative Commons Attribution 4.0 International License.

Read Full License 


\section{Abstract}

Objective: Gastric ancer is a common cancer with high cancer-related death in the world Although, ASPN has been reported as a potential biomarker in some cancers, the relationship of ASPN and clinical pathologic features in gastric cancer has not been investigated thoroughly. Thus, the aim of study is to evaluate the function of ASPN in gastric cancer based on TCGA.

Materials and methods: The gene expression data (407 cases, Workflow Type: HTSeq-Counts) and corresponding clinical information were downloaded from the TCGA Genomic Data Commons data portal. We performed the NES and nominal $p$ value to evaluate the pathways enriched in each phenotype. Then, we analyzed the association with ASPN and clinicopathologic variables based on Wilcoxon signedrank test and logistic regression.

Result: We found that it was significantly different in ASPN expression between gastric cancer and normal tissue $(P<0.001)$. High ASPN expression was significantly associated with high stage (Odds ratio $(O R)=3.656$ for stage II vs stage I and $O R=0.014$ for stage III vs stage I), T classification (OR=13.304 for $\mathrm{T} 2$ vs $\mathrm{T} 1, \mathrm{OR}=20.769$ for $\mathrm{T} 3$ vs $\mathrm{T} 1$ and $\mathrm{OR}=24.857$ for $\mathrm{T} 4$ vs $\mathrm{T} 1$ ) (all $\mathrm{p}$-values< 0.05 ). Univariate analysis revealed ASPN could result in poor overall survival with $H R=1.004$ and $P=0.036$. Besides, multivariate analysis indicated that ASPN expression was an independent risk factor for overall survival (HR:1.010, $P=0.000)$, age (HR: 1.046, $P=0.002)$ and gender (HR: 1.623, $P=0.026)$. Besides, GSEA results showed that Pentose phosphate pathway, Base excision repair, Peroxisome, Protesome, Nucleotide excision repair, and Mismatch repair were differentially enriched in gastric cancer with high ASPN expression phentype.

Conclusion: ASPN expression is higher in gastric cancer than normal tissues, and considered ASPN as a potential independent molecular marker for diagnosis and prognosis of GC.

\section{Introduction}

Gastric cancer (GC) is one of the most common cancer $n$ malignant tumors in the digestive system and remains the high cause of cancer-related death following lung and liver cancer in the world[1]. It is one of the most common malignant tumors in digestive system[2]. The people in Asia and Eastern Europe is suspetible to the gastric cancer, particular in China, and Middle and South America is also a comon certain areas of gastric cancer. There are differences in the occurence of disease in differernt geographic areas, with remarkly higher incidences of gastric cancer in local populace[3]. The location of occurence of gastric cancer is alomost mainly in the gastric antrum cancer and gastric carcinoma, and there is a gradually increase of gastroesophageal junction carcinoma[4]. Although the exact cause of gastric cancer is unclear, abnormal molecular mechanism and signaling pathways is associated with the poor prognosis and survivlal rate in cancer. It is reported that the prognostic value of some molecules were performed to evaluate the screening and diagnosis of gastric cancer $[5,6]$. Therefore, we should focus more attention on novel tumour markers with sufficient sensitivity and specificitly to promote the early diagnosis and prognostic prediction in gastric cancer patients. 
Asporin (ASPN) belongs to the small leucine-rich proteoglycan (SLRP) family and serves important roles in diverse biological responses and disease conditions. ASPN could promote proliferation and colony formation in gastric cancer cell lines[7] Besides, it could promote gastric cancer cell proliferation by interacting with proteasome $26 \mathrm{~S}$ subunit non-ATPase 2 (PSMD2) and then promoting the degradation of downstream effectors, which may play an important role in the tumor microenvironment of certain cancers. Ding et al[8] suggested that ASPN could enhance the growth and migration of cancer cell by regulating epidermal growth factor receptor (EGFR) signaling and the apoptosis pathway in gastric cancer. ASPN secreted by cancer-associated fibroblasts (CAFs) or cancer cells could promote the invasion and migration of cancers by regulating the epithelial to mesenchymal transition (EMT) or interacting with cell-membrane receptors[9-11]. It is reporeted reported that ASPN as the hub genes asporin were demonstrated to have prognostic value for patients with gastric cancer.[5] Liu et al[12] also reported that ASPN was constructed with a good performance in predicting overall survivals based on integrated bioinformatics analysis. ASPN in peripheral blood samples of patients with the disease suggest that expression profile of candidate gene could be used as a biomarker for predicting the development and progression of knee OA[13]. Ahthough, the previous study showed that ASPN may be a carcinogenic effect in gastric cancer based on bioinformatic analysis, and expression of ASPN predicts

favorable results in gastric cancer patients[5, 7]. However, the actions of ASPN in clinical significance and prognostic of gastric cancer is unclear.

In recent years, The Cancer Genome Atlas (TCGA), the application of high-throughput platforms, coutribute very valuable in the evaluation of diagnosis and prognosis of cancer in clinical research. In this study, we will explore the clinical diagnosis and prognosis value of ASPN in gastric cancer according to TCGA database. And then, we also further evaluate signalling pathways of gastric cancer pathogenesisassociated ASPN regulatory networks based on Gene Set Enrichment Analysis (GSEA).

\section{Materials And Methods}

\section{RNA-sequencing patient data and bioinformatics analysis}

The gene expression data (407 cases, Workflow Type: HTSeq-Counts) and corresponding clinical information were downloaded from the TCGA Genomic Data Commons data portal (https://portal.gdc.cancer.gov/repository). Those patients included 32 normal samples and 375 gastric cancer patients. RNA-Seq gene expression level 3 HTSeq-Counts data for adenomas and adenocarcinomas gastric cancer were downloaded and used for further analysis..

\section{Gene Set Enrichment Analysis}

GSEA is a computational method that determines whether an a priori defined set of genes shows statistically significant, concordant differences between two biological states[14]. In this study, we uploaded the datasets and phenotype label files associated with ASPN into GSEA software. ASPN-high 
and ASPN-low groups of phenotype label files were analyzed in GSEA. It is 1000 times for each analysis of ASPN in gene get permutations. We performed the normalized enrichment score (NES) and nominal $p$ value to evaluate the pathways enriched in each phenotype. Gene sets with a normal P-value $<0.05$ and false discovery rate $(F D R)<0.25$ ere used to sort the pathways enriched in each phenotype.

\section{Statistical analysis}

R software (v.3.6.1) was uesd to analyze all gene data. Besides, Wilcoxon signed-rank test and logistic regression were used to evaluate the relationship betweens ASPN expression and clinical pathologic features. Cox regression and the Kaplan-Meier method were used to explore clinicopathologic factors related to overall survival in ASPN expression of gastric cancer. Multivariate Cox analysis was performed to evaluate the action of ASPN expression on survival along with other clinical factors including age, gender, grade, tumor grade, T, N (lymph node metastasis) and $\mathrm{M}$ (Tumor metastasis). The median value was used to determine the the cutoff value of ASPN expression. Besides, High-ASPN expression and lowASPN expression was evaluated according to the median values.

\section{Results}

\section{Patient characteristics}

As in showed in Table 1, there were 241 (64.26\%) males and 134 (35.74\%) females with the median age at diagnosis of 65.69 years. The survival time was 504.17 days according to patients with gastric cancer. There were 10 cases $(2.67 \%)$ in Grade1, 136 cases (36.26\%) in Grade 2, 220 cases (58.67\%) in Grade 3 and 9 cases (2.40\%) in Grade X. There were 52 patients (13.87\%) in Stage I, 114 (30.40\%) patients in Stage II, 149 (39.73\%) patients in Stage III and 38 (10.13\%) patients in Stage IV, and 22 (5.87\%) patients was unknown. 112 (29.87\%) of 375 patiens had lymph node metastases (Pelvic and Para-aortic). 45 $(12.00 \%)$ of 375 patients had distant metastases. 
Table 1

Patient characteristics of gastric cancer in TCGA database

\begin{tabular}{|c|c|c|c|}
\hline \multicolumn{2}{|c|}{ Clinical characteristics } & Total(375) & $\%$ \\
\hline \multicolumn{2}{|l|}{ Age at diagnosis (y) } & \multicolumn{2}{|l|}{ 65.69(Year) } \\
\hline \multicolumn{2}{|l|}{ survival time } & \multicolumn{2}{|l|}{ 504.17(Day) } \\
\hline \multirow[t]{2}{*}{ Gender } & Male & 241 & 64.26 \\
\hline & Female & 134 & 35.74 \\
\hline \multirow[t]{2}{*}{ Survival status } & Live & 244 & 65.06 \\
\hline & dead & 131 & 34.94 \\
\hline \multirow[t]{4}{*}{ Grade } & G1 & 10 & 2.67 \\
\hline & $\mathrm{G} 2$ & 136 & 36.26 \\
\hline & G3 & 220 & 58.67 \\
\hline & $\mathrm{GX}$ & 9 & 2.40 \\
\hline \multirow[t]{5}{*}{ Stage } & Stage I & 52 & 13.87 \\
\hline & Stage II & 114 & 30.40 \\
\hline & Stage III & 149 & 39.73 \\
\hline & Stage IV & 38 & 10.13 \\
\hline & Unkonwn & 22 & 5.87 \\
\hline \multirow[t]{3}{*}{ Lymph nodes } & Negative & 261 & 69.60 \\
\hline & Positive & 112 & 29.87 \\
\hline & Unkonwn & 2 & 0.53 \\
\hline \multirow[t]{2}{*}{ Distant metastasis } & Negative & 330 & 88.00 \\
\hline & Positive & 45 & 12.00 \\
\hline
\end{tabular}

\section{Association With Aspn Expression And Clinicopathologic Variables}

According to the Fig. 1, there was significantly different in ASPN expression between gastric cancer and normal tissue $(P<0.001)$. The expression of ASPN in gastric cancer were correlated significantly with clinical stage $(P<0.001)$, histological stage $(P<0.001)$ and T classification $(P<0.001)$. Besides, there was no significantly different in ASPN expression wth clinical factors including age, gender, $\mathrm{M}$ and $\mathrm{N}$ classification $(P>0.05)$. 
The logistic regression of ASPN expression showed that it was associated with clinical pathological characteristics.(Table 2) There was remarkable relationship bewteen ASPN expression and stage classification (stage II vs stage I, OR $=3.656, P<0.001$ and stage III vs stage $\mathrm{I}, \mathrm{OR}=2.313, \mathrm{P}=0.014$ ) and $T$ classification ( $T$ classification $T 2$ vs $T 1, O R=13.304, P=0.014 ; T 3$ vs $T 1, O R=20.769, P=0.003$; and $\mathrm{T} 4 \mathrm{vs} T 1, \mathrm{OR}=24.857, \mathrm{P}=0.002$ ). The ASPN expression in gastric cancer was not remarkably related with age, gender, grade (G2 vs G1 and G3 vs G1), N classification (N1 + N2 + N3 vs N0) and M classification (M1 vs M0). The results showed that the expression of ASPN could indicate the stage classification and Lymph nodes metastasis of cancer.

Table 2

ASPN expression associated with clinical pathological characteristics (logistic regression)

\begin{tabular}{|lllll|}
\hline Clinical characteristics & $\begin{array}{l}\text { Total } \\
\text { (N) }\end{array}$ & $\begin{array}{l}\text { Odds ratio in ASPN } \\
\text { expression }\end{array}$ & 95\%Cl & p-Value \\
\hline Age & 371 & 1.013 & $0.670-1.531$ & 0.951 \\
\hline Gender & 375 & 0.903 & $0.591-1.379$ & 0.638 \\
\hline Grade (G2 vs G1) & 147 & 0.421 & $0.103-1.541$ & 0.195 \\
\hline Grade (G3 vs G1) & 229 & 0.870 & $0.217-3.131$ & 0.833 \\
\hline Stage classification (II vs I) & 164 & 3.656 & $1.843-7.517$ & 0.000 \\
\hline Stage classification (III vs & 203 & 2.313 & $1.203-4.607$ & 0.014 \\
\hline I) & & & & $0.787-4.505$ \\
\hline Stage classification (IV vs & 91 & 1.872 & $2.551-$ & 0.157 \\
\hline I) & & & 245.163 & 0.014 \\
\hline T classification (T2 vs T1) & 99 & 13.304 & $4.142-$ & 0.003 \\
\hline T classification (T3 vs T1) & 187 & 20.769 & 377.928 & \\
\hline T classification (T4 vs T1) & 119 & 24.857 & $4.839-$ & 0.002 \\
\hline N classification & 357 & 0.743 & $0.472-1.164$ & 0.196 \\
\hline (N1 + N2 + N3vsN0) & & & $0.335-1.755$ & 0.544 \\
\hline M classification(M1 vs M0) & 355 & 0.776 & & \\
\hline
\end{tabular}

\section{Univariate And Multivariate Analysis Of Aspn}

As was showed in Table 3, high ASPN exprssion in univariate analysis was significantly associated with poor overall survival (HR:1.004, 95\% Cl:1.000-1.009 and $\mathrm{P}=0.036)$. Besides, multivariate Cox analysis indicated that high ASPN expression was significantly associated overall survival (HR:1.010, 95\% Cl: 
$1.005-1.015, \mathrm{P}=0.000)$, age (HR: $1.046,95 \% \mathrm{Cl}: 1.024-1.068, \mathrm{P}=0.002)$ and gender (HR: $1.623,95 \% \mathrm{Cl}$ : $1.057-2.492, P=0.026)$. There were other clinical factors associated with clinical factors including Grade, Stage classification, and TNM classification.

Table 3

The relationship between ASPN expression and overall survival in GC patients based on univariate and multivariate analysis.

\begin{tabular}{|llll|}
\hline Clinicopathologic variable & HR & $95 \% \mathrm{Cl}$ & P-value \\
\hline a. Univariate analysis & & & \\
\hline ASPN & 1.004 & $1.000-1.009$ & 0.036 \\
\hline b. Multivariate analysis & & & \\
\hline Age & 1.046 & $1.024-1.068$ & 0.002 \\
\hline Gender & 1.623 & $1.057-2.492$ & 0.026 \\
\hline Grade & 1.310 & $0.889-1.929$ & 0.171 \\
\hline Stage classification & 1.485 & $0.960-2.298$ & 0.075 \\
\hline T classification & 1.032 & $0.747-1.426$ & 0.844 \\
\hline M classification & 2.018 & $0.916-4.449$ & 0.081 \\
\hline N classification & 1.092 & $0.854-1.396$ & 0.482 \\
\hline ASPN & 1.010 & $1.005-1.015$ & 0.000 \\
\hline HR: hazard ratio; Cl: confidence interval. & \\
\hline
\end{tabular}

\section{Aspn-associated Signaling Pathways By Gsea}

In order to evaluate the potential signaling pathways associated with ASPN in gastric cancer, we conducted the high and low ASPN expression datasets by Gene Set Enrichment Analysis (GSEA). GSEA showed the remarkably differences in the enrichment of MSigDB collection

(c2.cp.kegg.v6.2.symbols.gmt), which was a collection of annotated gene sets for use with GSEA software. The important signaling pathways associated with ASPN were identified according to KEGG pathway analysis. In high expression datasets of ASPN, 72 gene sets were significant at FDR $<25 \%$, and 22 gene sets were significantly enriched at nominal pvalue $<1 \%$. In low expression datasets of ASPN, 60 gene sets were significantly enriched at FDR $<25 \%$ and 23 gene sets were significantly enriched at nominal pvalue $<1 \%$. GSEA showed that Pentose phosphate pathway (a), Base excision repair (b), Peroxisome (c), Protesome (d), Nucleotide excision repair (e), and Mismatch repair (f) were differentially enriched in ASPN high expression phenotype in gastric cancer (FDR < 0.05, NOM p-val <0.05) (Fig. 2). Focal adhesion, Calcium signaling pathway, Ecm receptor interaction, TGF- $\beta$ signaling pathway, Calcium Signaling pathway and Hedgehog signaling pathway. were differentially enriched in ASPN low expression 
phenotype in gastric cancer (FDR < 0.001, NOM p-val < 0.01). We selected the 6 high and 6 low expression enriched signaling pathways of KEGG in Table 4 and Fig. 3.

Table 4

Gene sets enriched in phenotype high and low expression of ASPN based on gene set enrichment analysis.

\begin{tabular}{|lllll|}
\hline Gene set name & ES & NES & NOM p-val & FDR q-val \\
\hline a. High expression datasets of ASPN & & & & \\
\hline KEGG_PENTOSE_PHOSPHATE_PATHWAY & 0.7245 & 2.1051 & 0 & 0.0116 \\
\hline KEGG_BASE_EXCISION_REPAIR & 0.7917 & 2.1503 & 0 & 0.0096 \\
\hline KEGG_PEROXISOME & 0.6260 & 2.0217 & 0.0019 & 0.0103 \\
\hline KEGG_PROTEASOME & 0.8162 & 2.0542 & 0 & 0.0118 \\
\hline KEGG_NUCLEOTIDE_EXCISION_REPAIR & 0.6677 & 1.8890 & 0.0122 & 0.0246 \\
\hline KEGG_MISMATCH_REPAIR & 0.8210 & 1.9923 & 0 & 0.0110 \\
\hline b. Low expression datasets of ASPN & & & & 0 \\
\hline KEGG_FOCAL_ADHESION & -0.7179 & -2.3743 & 0 & 0 \\
\hline KEGG_CALCIUM_SIGNALING_PATHWAY & -0.6001 & -2.2732 & 0 & 0 \\
\hline KEGG_ECM_RECEPTOR_INTERACTION & -0.811 & -2.3963 & 0 & 0.0021 \\
\hline KEGG_BASAL_CELL_CARCINOMA & -0.6716 & -2.13749 & 0 & 0.0054 \\
\hline KEGG_TGF_BETA_SIGNALING_PATHWAY & -0.6014 & -2.03032 & 0 & 0.0019 \\
\hline KEGG_HEDGEHOG_SIGNALING_PATHWAY & -0.6688 & -2.1465 & 0 & \\
\hline NES: normalized enrichment score; NOM: nominal; FDR: false discovery rate. & \\
\hline Gene sets with NOM p-val < 0.05 and FDR q-va < 0.25 are considered as significant. \\
\hline
\end{tabular}

\section{Discussion}

Gastric cancer is one of the most common tumour of gastroenteric tumor in the world. China is the high incidence areas of gastric cancer, which could influence the lifetime of human[15, 16]. The advanced histological stage or distant metastasis could cause the poor prognosis of gastric cancer. Therefore, early diagnosis or surgery is significantly important to the treatment of tumor. In those studies, many scholar explored the novel biomarkers for early diagnosis or evaluation the prognosis and recurrence in gastric cancer [17-19]. TFAP-2 $\beta$ constituted promoter activity in gastric cancer and contributed to the development of the metabolic syndrome. TFAP- $2 \beta$ may influence the occurrence and development of gastric through regulating the expression of various adipokines and lipoprotein metabolism[14, 20]. Li et 
al reported that high VCAN was expressed in gastric cancer and considered it as a independent molecular marker for diagnosis and prognosis of gastric cancer[21].

ASPN,a member of small leucine-rich repeat proteoglycan (SLRP) family of proteins, is localized on chromosome 9q22.31 and encodes a secretory protein containing 380 amino acids[7]. It could focus on the diverse biological responses and disease conditions. In previous studies, many authors suggested that ASPN was significantly associated with the development of various types of cancer $[7,10,22]$. For example, Bàrbara et al [9] showed that ASPN and GJB2 could take part in the mechanism of local invasion in breast carcinomas. Maris et al [23] also reporetd that asporin, as a stroma-derived inhibitor of TGF- $\beta 1$, could suppress the development of tumors and reduce the aggressive of breast cancer in clinical results. Besiedes, cancer-associated fibroblasts or cancer cells could secreted ASPN to regulate the epithelial to mesenchymal transition or interact with cell-membrane receptor, which could promote the invasion and migration of tumor[7]. Satoyoshi et al[24] showed that asporin could activate coordinated invasion of cancer-associated fibroblasts and scirrhous gastric cancer and promote the progression disease of tumour cells. The expression of asporin could bring a novel therapeutic molecular target for the diagnosis and treatment of gastric cancer. However, there was no reported to identify the realtionship between ASPN and clinical evaluation in gastric cancer.

In this study, we found that there was significantly different in ASPN expression between gastric cancer and normal tissue $(P<0.001)$. The expression of ASPN was markedly higher in gastric cancer compared with normal tissues. The result was similar with previous studies. The bioinformatic analysis also showed that increased ASPN expression in gastric cancer compared with adjacent normal gastric according to the high throughput RNA-sequencing data[5, 12]. Ding et al[8] reported that ASPN was overexpressed in gastric carcinoma tissues when compared to the corresponding noncancerous tissues. We summarized that the expression of ASPN in gastric cancer was significantly associated with clinical stage $(P<0.001)$ histological stage $(P<0.001)$ and $T$ classification $(P<0.001)$, which indicated that ASPN may regulate the above clincial factors. In Jiang et al study, overall survival and diease free survival in KaplanMeier curves showed that high expression of ASPN caused the poor prognosis in GC patients $(P<0.05)[5]$.

Besides, logistic regression showed that ASPN expression was remarkable associated with stage classification (stage II vs stage I, OR $=3.656, \mathrm{P}<0.001$ and stage III vs stage $\mathrm{I}, \mathrm{OR}=2.313, \mathrm{P}<0.0141$ ) and $T$ classification ( $T$ classification $T 2$ vs $T 1, O R=13.304, P=0.014 ; T 3$ vs $T 1, O R=20.769, P=0.003$ and $T 4$ vs $T 1, O R=24.857, P=0.002$ ). Those results showed that ASPN could promote the stage classification and Lymph nodes metastasis. Therefore, we hypothesized that ASPN could be potential therapeutic targets in the molecular mechanism for treatment of GC. The univariate analysis showed that increase exprssion of ASPN was remarkably associated with poor overall survival (OR:1.005, 95\% Cl:1.000-1.009 and $P=0.036$ ). The multivariate Cox analysis indicated that high VCAN expression was significantly associated overall survival (HR:1.010, 95\%Cl: 1.005-1.015, P =0.000), age (HR: $1.046,95 \% \mathrm{Cl}: 1.024-$ $1.068, \mathrm{P}=0.002$ ) and gender (HR: 1.623, 95\%Cl: 1.057-2.492, $\mathrm{P}=0.026)$. In previous study, the author considered ASPN as an important molecular gene in the developmeng of cancer to contribute the 
progression and metastasis via the epidermal growth factor receptor (EGFR) signalling pathway ${ }^{[8]}$. ASPN promotes the proliferation of GC cells by interacting with PSMD2, and knockdown of ASPN significantly increased the expression of dual specificity phosphatase 7(DUSP7), which served as a potential therapeutic target in gastric cancer [7].

We performed the high and low ASPN expression datasets by GSEA in the enrichment of the MSigDB collection (c2.cp.kegg.v6.2.symbols.gmt). We reported that GSEA showed that Pentose phosphate pathway, Base excision repair, Peroxisome, Protesome, Nucleotide excision repair, and Mismatch repair were differentially enriched in ASPN high expression phenotype in gastric cancer (FDR $<0.05$, NOM $p$-val $<0.05$ ). Those results was concordant with previous studies[25]. In gastric cancer, there were DNA repair systems of cancer cells could maintain the integrity and stability of the genome including base excision repair, mismatch repair,nucleotide excision repair and double strand break repair[26]. Pentose phosphate pathway play an important part in cellular metabolism, ribonucleotide and lipid biosynthesis to promote the survival of cancer cells, which sustain antioxidant responses to support cell survival and proliferation[27]. Besides, it was conducted to maintain cancer cells in anabolic demands and redox homeostasis, and cancer cells acquired multiple mechanisms to deregulate the oxidative and nonoxidative pentose phosphate pathway[28, 29]. Choi et al[30] reported that expression of pentose phosphate pathway was higher in breast cancer compared with normal tissue according to molecular subtype. Pentose phosphate pathway was significantly expressed in gastric cancer tissue with low expression of Rev-erba, which resulted in the progession of gastric cancer[31]. Base excision repair, the most prevalent pathway in damaged bases modification, could repair the vast majority of endogenous DNA damage including alkylations, oxidations, deaminations and depurinations, as well as single-strand breaks in cancer cells[32]. Biological function of BER removed these frequently produced lesions, maintained genomic integrity and affected the clinical prognosis and overall survival of gastric cancer according to the promotion of DNA repair capacity[33]. In previous study, the aurthor could regulate the base excision repair system to inhibite the growth of gastric cancer and provide the novel perspectives to evluate gastric cancer progression based on the role of base excision repair[34].

Focal adhesion, Calcium signaling pathway, Ecm receptor interaction, TGF- $ß$ signaling pathway, Calcium Signaling pathway and Hedgehog signaling pathway. were differentially enriched in ASPN low expression phenotype in gastric cancer (FDR $<0.001$, NOM p-val $<0.01$ ). In previous study, the enrichment analysis in gastric cancer were enriched in ECM-receptor interaction, focal adhesion, metabolism of xenobiotics and drug metabolism pathways, and the result was similar with our study[5]. Cao et al[35] also analyzed that KEGG pathways of differentially expressed genes were significantly enriched in ECM-receptor interaction, protein digestion and absorption, and the focal adhesion pathways, and the genes in the modules of protein-protein interaction were mainly involved in the ECM-receptor interaction and focal adhesion pathways. The activation of hedgehog signaling pathway could promote the neoplastic transformation and the developmeng of gastric cancer by the biological function of cancer stroma interaction[36].Therefore, we could monitor Hedgehog signaling in order to evlauated the eventually developing gastric cancer. And then, Upregulation of Hedgehog signaling pathway was associated with 
tumor development. Hedgehog-independent activation of Patched through the action of proteases and in particular Caspase 3, splitting the C-terminal from Patched[37, 38].

In conclusion, according to the TCGA databases, we considered ASPN expression as a potential molecular marker in the diagnosis and prognostic of overall survival in gastric cancer. Furthermore, the pentose phosphate pathway, base excision repair, peroxisome, protesome, nucleotide excision repair, and mismatch repair may be the remarkable key pathway regulated by ASPN in based on the GSEA anaysis software.

\section{Abbreviations}

ASPN:Asporin; GC: gastric cancer; TCGA: The Cancer Genome Atlas; OR: Odds ratio; HR: Hazard ratio; SLRP: Small leucine-rich proteoglycan; PSMD2: Proteasome 26S subunit non-ATPase 2; EGFR: Epidermal growth factor receptor; CAFs: Cancer-associated fibroblasts; EMT: Epithelial to mesenchymal transition; OA: Osteoarthritis; GSEA: Gene Set Enrichment Analysis; NES: normalized enrichment score; FDR: False discovery rate; KEGG:Kyoto Encyclopedia of Genes and Genomes; SLRP: small leucine-rich repeat proteoglycan.

\section{Declarations}

\section{Ethics approval and consent to participate}

The study protocol was approved by the Research Ethics Committee of Seven Medical Center of Chinese People's Liberation Army General Hospital.

\section{Availability of data and materials}

The datasets used and analysed during the current study are available from the corresponding author on reasonable request.

\section{Competing interests}

The authors declare that they have no competing interests" in this section.

\section{Funding}

Not applicable.

\section{Authors' contributions}

W.C.L and R.J.X contributed to the conception and design. W.C.L and H.C were responsible for the drafting of the manuscript. Z.D.W and Y.X were responsible for the data acquisition and analysis of data. Z.X and L.L contributed to the collection and assemblyof data. W.C.L provided professional writing services for papers. All authors read and approved the final manuscript. 
Acknowledgements

Not applicable.

\section{Competing interests}

The authors have declared that no competing interest exists.

\section{References}

1. Ferlay J, Soerjomataram I, Dikshit R, et al: Cancer incidence and mortality worldwide: sources, methods and major patterns in GLOBOCAN 2012. International journal of cancer 2015, 136(5):E359386.

2. Song Z, Wu Y, Yang J, et al. Progress in the treatment of advanced gastric cancer. Tumour biology: the journal of the International Society for Oncodevelopmental Biology Medicine. 2017;39(7):1010428317714626.

3. Arnold M, Moore SP, Hassler S, et al. The burden of stomach cancer in indigenous populations: a systematic review and global assessment. Gut. 2014;63(1):64-71.

4. Lee HS, Kim WH, Kwak Y, et al: Molecular Testing for Gastrointestinal Cancer. Journal of pathology and translational medicine 2017, 51(2):103-121.

5. Jiang K, Liu H, Xie D, et al. Differentially expressed genes ASPN, COL1A1, FN1, VCAN and MUC5AC are potential prognostic biomarkers for gastric cancer. Oncology letters. 2019;17(3):3191-202.

6. Zha Y, Cun Y, Zhang Q, et al. Prognostic value of expression of Kit67, p53, Topolla and GSTP1 for curatively resected advanced gastric cancer patients receiving adjuvant paclitaxel plus capecitabine chemotherapy. Hepato-gastroenterology. 2012;59(117):1327-32.

7. Zhang Z, Li H, Zhao Y, et al. Asporin promotes cell proliferation via interacting with PSMD2 in gastric cancer. Frontiers in bioscience. 2019;24:1178-89.

8. Ding Q, Zhang M, Liu C. Asporin participates in gastric cancer cell growth and migration by influencing EGF receptor signaling. Oncol Rep. 2015;33(4):1783-90.

9. Castellana B, Escuin D, Peiro G, et al. ASPN and GJB2 Are Implicated in the Mechanisms of Invasion of Ductal Breast Carcinomas. J Cancer. 2012;3:175-83.

10. Wang $L, W u H$, Wang $L$, et al. Asporin promotes pancreatic cancer cell invasion and migration by regulating the epithelial-to-mesenchymal transition (EMT) through both autocrine and paracrine mechanisms. Cancer letters. 2017;398:24-36.

11. Hurley PJ, Sundi D, Shinder B, et al. Germline Variants in Asporin Vary by Race, Modulate the Tumor Microenvironment, and Are Differentially Associated with Metastatic Prostate Cancer. Clinical cancer research: an official journal of the American Association for Cancer Research. 2016;22(2):448-58.

12. Liu X, Wu J, Zhang D, et al: Identification of Potential Key Genes Associated With the Pathogenesis and Prognosis of Gastric Cancer Based on Integrated Bioinformatics Analysis. Frontiers in genetics 
2018, 9:265.

13. Mishra A, Awasthi S, Raj S, et al. Identifying the role of ASPN and COMP genes in knee osteoarthritis development. J Orthop Surg Res. 2019;14(1):337.

14. Wu H, Zhang J. Decreased expression of TFAP2B in endometrial cancer predicts poor prognosis: A study based on TCGA data. Gynecol Oncol. 2018;149(3):592-7.

15. Sugita $H$, lida $S$, Inokuchi $M$, et al. Methylation of BNIP3 and DAPK indicates lower response to chemotherapy and poor prognosis in gastric cancer. Oncol Rep. 2011;25(2):513-8.

16. Kim YI, Choi IJ, Lee JY, et al. Comparison of the performance of risk scoring systems for tumor bleeding in patients with inoperable gastric cancer. Endoscopy; 2020.

17. Noh G, Kim N, Choi Y, et al: Long-term follow-up of serum pepsinogens in patients with gastric cancer or dysplasia after Helicobacter pylori eradication. Journal of gastroenterology and hepatology 2020.

18. Son HJ, Choi EJ, Yoo NJ, et al: Mutational and expressional alterations of a candidate tumor suppressor HECA gene in gastric and colorectal cancers. Pathology, research and practice 2020:152896.

19. Sun T, Wang D, Ping Y, et al: Integrated profiling identifies SLC5A6 and MFAP2 as novel diagnostic and prognostic biomarkers in gastric cancer patients. International journal of oncology 2020, 56(2):460-469.

20. Cui P, Shi K, Cui HX, et al: Correlation between transcription factor activator protein-2beta (TFAP2beta) and endometrial carcinoma. European journal of gynaecological oncology 2015, 36(3):268273.

21. Li W, Han F, Fu M, et al. High expression of VCAN is an independent predictor of poor prognosis in gastric cancer. J Int Med Res. 2020;48(1):300060519891271.

22. Wu $H$, Jing $X$, Cheng $X$, et al. Asporin enhances colorectal cancer metastasis through activating the EGFR/src/cortactin signaling pathway. Oncotarget. 2016;7(45):73402-13.

23. Maris P, Blomme A, Palacios AP, et al. Asporin Is a Fibroblast-Derived TGF-beta1 Inhibitor and a Tumor Suppressor Associated with Good Prognosis in Breast Cancer. PLoS Med. 2015;12(9):e1001871.

24. Satoyoshi R, Kuriyama S, Aiba N, et al: Asporin activates coordinated invasion of scirrhous gastric cancer and cancer-associated fibroblasts. Oncogene 2015, 34(5):650-660.

25. Wood RD, Mitchell M, Sgouros J, et al: Human DNA repair genes. Science 2001, 291(5507):12841289.

26. He J, Zhuo ZJ, Zhang A, et al. Genetic variants in the nucleotide excision repair pathway genes and gastric cancer susceptibility in a southern Chinese population. Cancer management research. 2018;10:765-74.

27. Stincone A, Prigione A, Cramer T, et al. The return of metabolism: biochemistry and physiology of the pentose phosphate pathway. Biol Rev Camb Philos Soc. 2015;90(3):927-63. 
28. Patra KC, Hay N. The pentose phosphate pathway and cancer. Trends in biochemical sciences 2014 , 39(8):347-354.

29. Jiang P, Du W, Wu M. Regulation of the pentose phosphate pathway in cancer. Protein cell. 2014;5(8):592-602.

30. Choi J, Kim ES, Koo JS: Expression of Pentose Phosphate Pathway-Related Proteins in Breast Cancer. Disease markers 2018, 2018:9369358.

31. Tao L, Yu H, Liang R, et al: Rev-erbalpha inhibits proliferation by reducing glycolytic flux and pentose phosphate pathway in human gastric cancer cells. Oncogenesis 2019, 8(10):57.

32. Wallace SS. Base excision repair: a critical player in many games. DNA Repair. 2014;19:14-26.

33. Simonelli V, Leuzzi G, Basile G, et al: Crosstalk between mismatch repair and base excision repair in human gastric cancer. Oncotarget 2017, 8(49):84827-84840.

34. Ni F, Tang H, Wang C, et al. Berzosertib (VE-822) inhibits gastric cancer cell proliferation via base excision repair system. Cancer management research. 2019;11:8391-405.

35. Cao $L$, Chen $Y$, Zhang M, et al. Identification of hub genes and potential molecular mechanisms in gastric cancer by integrated bioinformatics analysis. PeerJ. 2018;6:e5180.

36. Akyala Al, Peppelenbosch MP. Gastric cancer and Hedgehog signaling pathway: emerging new paradigms. Genes \& cancer 2018, 9(1-2):1-10.

37. Thibert C, Teillet MA, Lapointe F, et al: Inhibition of neuroepithelial patched-induced apoptosis by sonic hedgehog. Science 2003, 301(5634):843-846.

38. Jenkins D, Winyard PJ, Woolf AS. Immunohistochemical analysis of Sonic hedgehog signalling in normal human urinary tract development. Journal of anatomy 2007, 211(5):620-629.

\section{Figures}



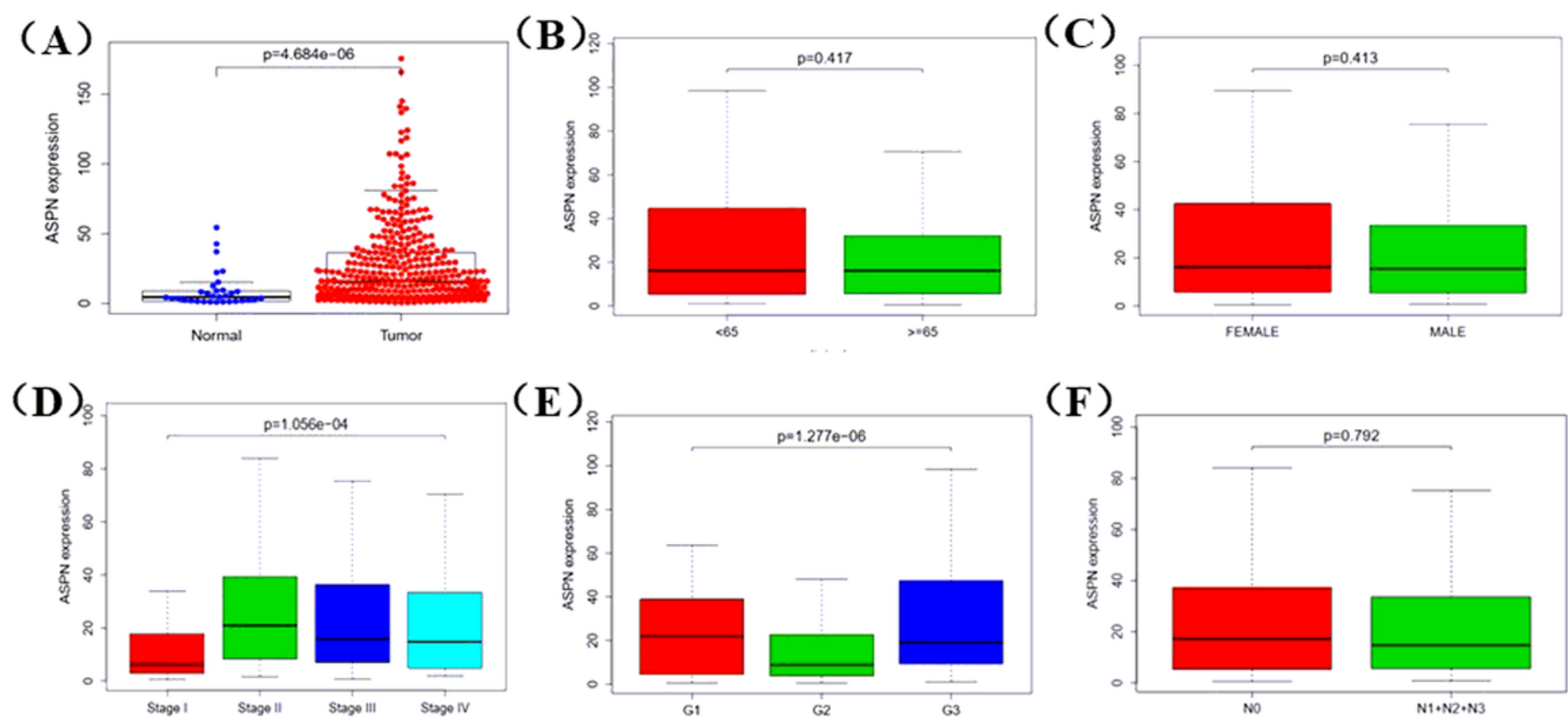

$($ E)

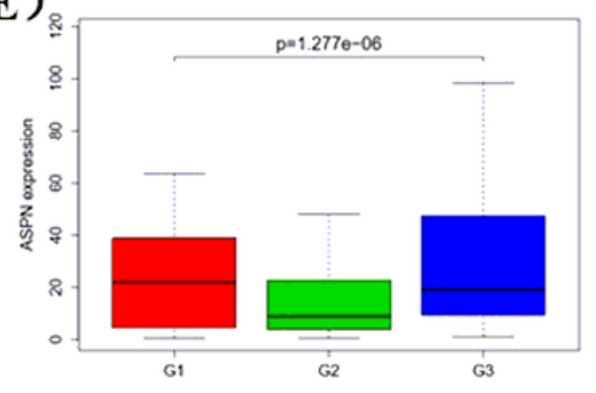

(F)

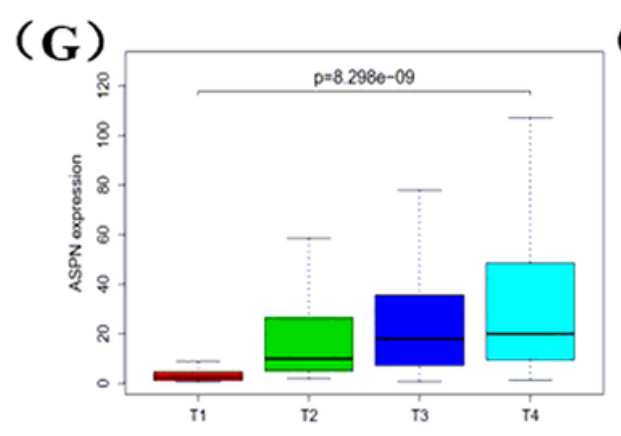

(H)
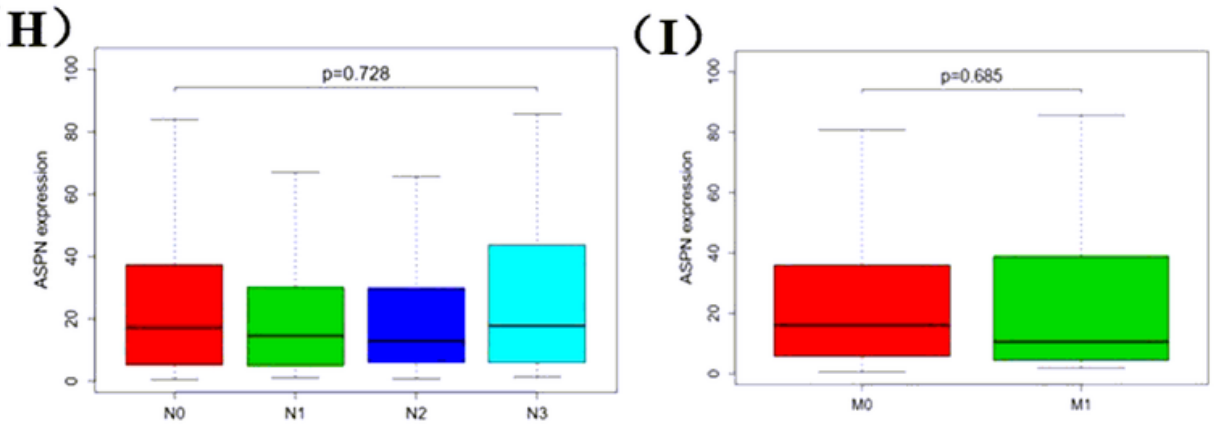

\section{Figure 1}

Association with ASPN expression with clinical characteristics. A:The expression level of ASPN in gastric cancer and normal tissues.; B.Age; C:Gender; D: Clinical stage; E: Grade; F: N classification of lymph node metastasis in (N1+N2+N3 vs N0); G:T classification; H: N classification of lymph node metastasis; I: M classification of distant metastases. 
(A) Enrichment plot:

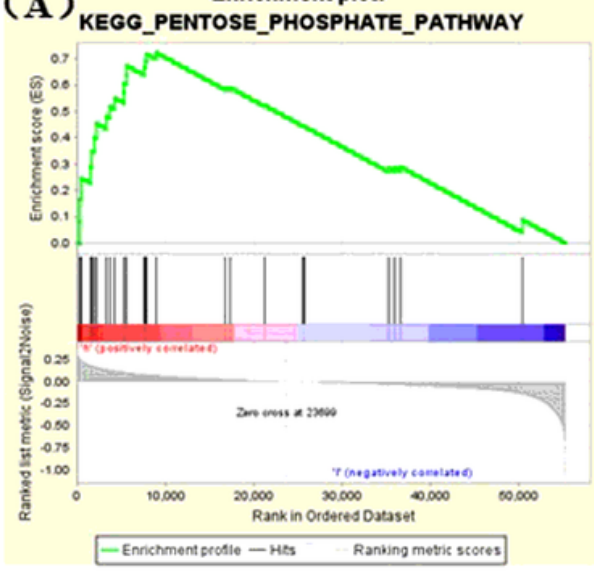

(D) Enrichment plot: KEGG_PROTEASOME

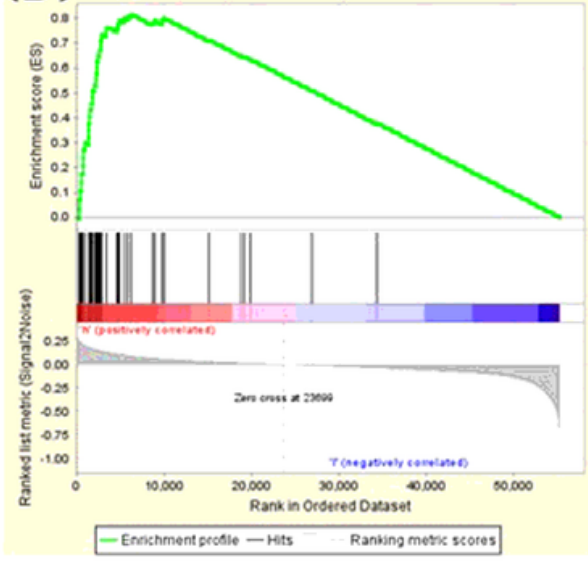

(Bin)chment plot: KEGG_BASE_EXCISION_REPAIR

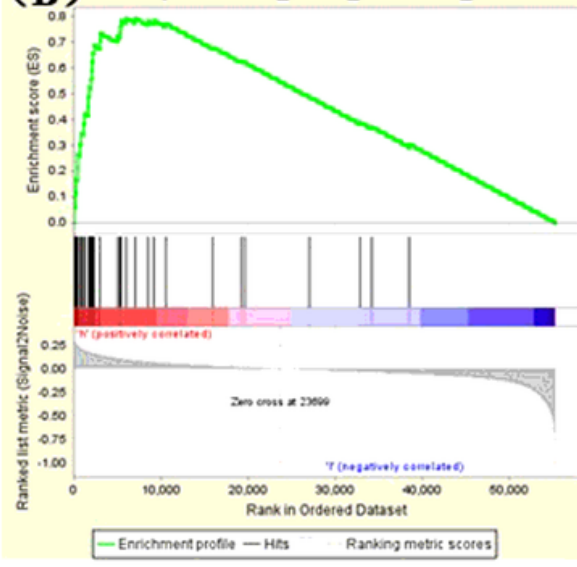

Enrichment plot:
(E)
KEGG_NUCLEOTIDE_EXCISION_REPAIR

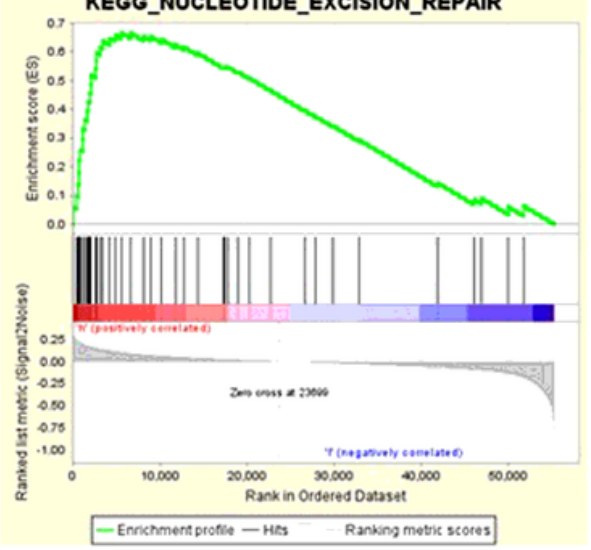

(C) Enrichment plot: KEGG_PEROXISOME

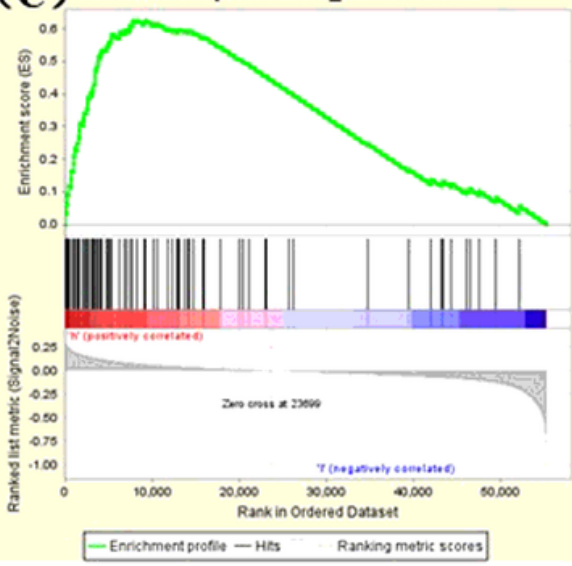

(F) Enrichment plot: KEGG_MISMATCH_REPAIR

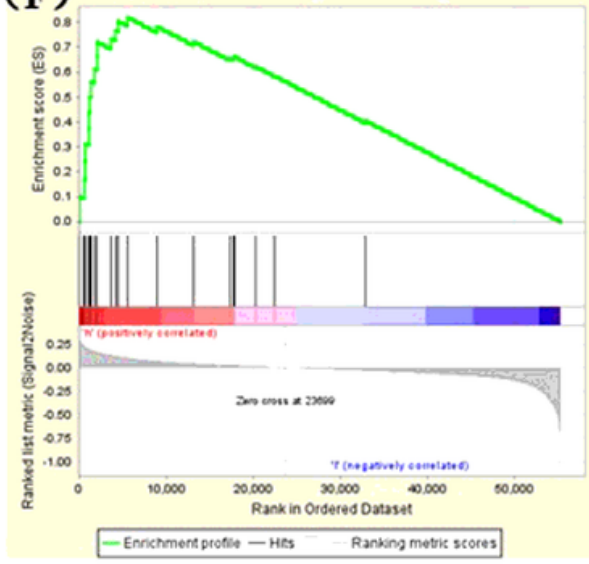

\section{Figure 2}

Enrichment plots from gene set enrichment analysis in GSEA. GSEA results showing Pentose phosphate pathway (A), Base excision repair (B), Peroxisome (C), Protesome (D), Nucleotide excision repair (E), and Mismatch repair ( $(F)$ were differentially enriched in ASPN associated gastric cancer 


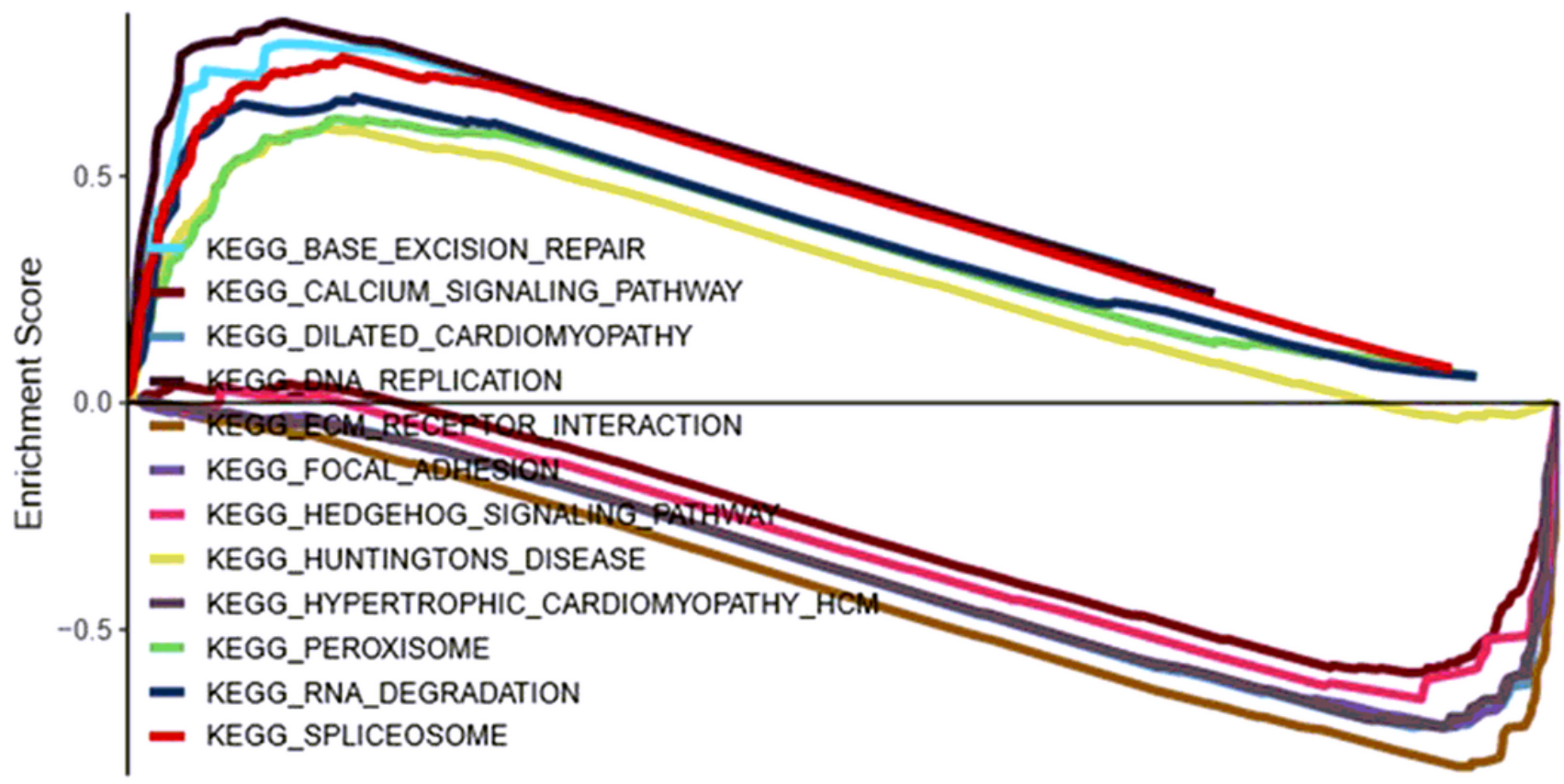

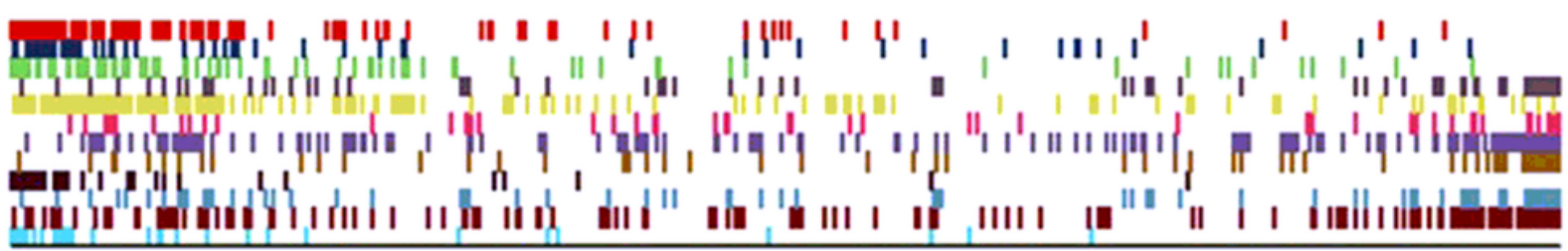

high expression<------------>low expression

Figure 3

Enrichment score for high and low expression of ASPN in enrichment analysis of GSEA 